\title{
A Pilot Study on the Addition of Tramadol or Eutectic Mixture of Local Anesthetics (Prilocaine Plus Lignocaine) to Local Lignocaine Infiltration for Prevention of Bone Marrow Aspiration/Biopsy Associated Pain
}

\author{
AH. Rudresha ${ }^{1}$ Bipinesh Sansar ${ }^{1}$ \\ D. Lokanath $1, \odot$ \\ Linu Abraham Jacob ${ }^{1}$ \\ M.C. Suresh Babu ${ }^{1}$ \\ Lokesh K. N. $^{1} \quad$ Smitha C. Saldanha ${ }^{1} \quad$ Shina Goyal ${ }^{1}$ \\ L. K. Rajeev ${ }^{1, \odot}$
}

${ }^{1}$ Department of Medical Oncology, Kidwai Memorial Institute of Oncology, Bengaluru, India

Address for correspondence Bipinesh Sansar, MD, DM, Department of Medical Oncology, Kidwai Memorial Institute of Oncology, Hombegowda Nagar, Bengaluru 560029, India

South Asian J Cancer 2021;10:190-194. (e-mail: bipinesh04@yahoo.co.in).

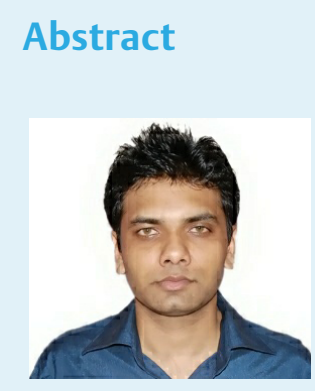

Bipinesh Sansar
Keywords

- aspiration

- bone marrow

- EMLA

- pain

- tramadol
Objectives Bone marrow aspiration although being a common procedure is associated with significant pain and its reduction remains an unmet need. We evaluated the use of tramadol and eutectic mixture of local anesthetics (prilocaine plus lignocaine) (EMLA) for reducing the severity of pain.

Materials and Methods In this pilot study, we compared the addition of either tramadol $50 \mathrm{mg}$ per oral (T) or EMLA local application (E) or no intervention (L) in addition to the usual procedure of local infiltration with lignocaine $2 \%$ before bone marrow aspiration and biopsy (BMAB) in adults suspected/confirmed with malignancy. Both, tramadol and EMLA were administered 1 hour prior to the procedure. Primary end point was reduction in pain intensity with these interventions compared with local infiltration alone. Pain was assessed using numerical FACES pain scale, a visual analogue scale. Secondary end points were to see the effect on pre procedure apprehension and to find out the other factors associated with increased pain related to the procedure.

Statistical Analysis and Results A total of 300 patients were included in the study, 100 each in tramadol (T), EMLA (E), and only lignocaine local infiltration (L) arms, respectively. The mean pain intensity on the visual scale was significantly lower in the tramadol arm (T, E, L-3.4, 4.4, 4.7, respectively) $(p<0.0005)$. There was a significant reduction in percentage of patients who experienced moderate/severe pain (four or more) in the tramadol arm (T, E, L-45, 77, 82\%, respectively) $(p<0.0005)$. Duration of procedure $>10$ minutes, body mass index $>30$, ECOG (Eastern Oncology Group) performance status $\geq 3$, and age $>50$ years were positively correlated with more pain. Leukemia patients experienced significantly more pain compared with patients with lymphoma and other solid malignancies. Tramadol was well tolerated. No significant effect on pre-procedure apprehension was noted in any of the arms.

Conclusion Tramadol appears to have a preventive effect on bone marrow aspiration/biopsy-associated pain and appears to be well tolerated, whereas EMLA was not associated with such an effect. Larger studies may be done to ascertain the same.
How to cite this article: Rudresha A. H, Sansar B, Lokanath D, et al. Pilot Study on the Addition of Tramadol or Eutectic Mixture of Local Anesthetics (Prilocaine Plus Lignocaine) to Local Lignocaine Infiltration for Prevention of Bone Marrow Aspiration/Biopsy Associated Pain. South Asian J Cancer 2021;10(3):190-194.
(C) 2021. MedIntel Services Pvt Ltd.

This is an open access article published by Thieme under the terms of the Creative Commons Attribution-NonDerivative-NonCommercial-License, permitting copying and reproduction so long as the original work is given appropriate credit. Contents may not be used for commercial purposes, or adapted, remixed, transformed or built upon. (https://creativecommons.org/licenses/by-nc-nd/4.0/).

Thieme Medical and Scientific Publishers Private Ltd A-12, Second Floor, Sector -2, NOIDA -201301, India 


\section{Introduction}

Bone marrow aspiration and biopsy (BMAB) is an integral part of the diagnosis and/or staging in various hematological malignancies. It is also essential to know the bone marrow involvement in solid malignancies which may have a bearing on further management. This useful procedure is associated with significant pain, the severity of which has been postulated to be affected by different factors. ${ }^{1}$ Local infiltration of the skin and periosteum at the site of the procedure with lignocaine has been used as the most accepted form of pre procedure analgesic of choice. ${ }^{2}$

Anxiety has been well known to intensify the pain during different medical procedures including BMAB. Patients undergoing BMAB are likely to be quite anxious and thus reducing anxiety may also serve to reduce the pain with BMAB. $^{3}$

Various studies have been done in adult and pediatric patients undergoing this procedure to evaluate the reduction in pain intensity with different interventions. These have included the use of inhalational anesthetics like nitrous oxide, analgesics like opioids, sedatives, and also anxiolytics like benzodiazepines or a combination of these. 4,5

But, despite that, there is no clear consensus whether any of these interventions provide added benefit and if they do, to what extent. This necessitates further trials with these agents in different populations and settings to clarify and to be able to start broad use of any of these interventions. ${ }^{6}$

Opioids have been used prophylactically for reducing pain before various procedures in some settings. Oral opioids like oxycodone and tramadol have been used in small populations and been found to be useful in significantly reducing the pain associated with this procedure in the adult population. ${ }^{4}$ Tramadol is a synthetic, centrally acting analgesic with dual mode of action. It is a weak mu opioid agonist and an inhibitor of noradrenaline and serotonin uptake. The peak analgesic effect starts between 1 and 4 hours and lasts 3 to 6 hours. It is widely available and well tolerated.

Eutectic mixture of local anesthetics 5\% (i.e., prilocaine 2.5\% plus lignocaine 2.5\%) (EMA) is the first topical anesthetic found to penetrate the intact skin and provide reliable anesthesia. This preparation has been used in children and adults for reducing pain associated with many minor procedures like lumbar punctures, etc., and is found to be useful. In children, it has been used with bone marrow aspiration/biopsy and found effective in reducing pain severity. ${ }^{7}$

In this study, we have attempted to use tramadol or EMA cream in addition to local infiltration of lignocaine respectively to assess whether these two have preventive effects on the pain and anxiety associated with BMAB. We have also analyzed the factors which may be associated with increased pain with $B M A B$ and how these interventions influence those factors.

\section{Patients and Methods}

This study was approved and cleared by the scientific review board and the Ethics Committee of our institute.
Eligible patients for this study were adult patients (more than 16 years of age) planned to undergo BMAB at our Centre for evaluation of malignancy and also repeat procedures for further evaluation and subsequently willing to give informed consent to participate in the study. Exclusion criteria included known hypersensitivity to tramadol or EMA, decompensated chronic liver, lung or kidney disease, history of seizures, chronic use of opioids, anticonvulsants, tricyclic antidepressants or monoamine oxidase inhibitors, pregnancy, and inability to interpret the questionnaire.

Patients eligible to participate in the study were randomized $1: 1: 1$, respectively into the three arms, viz: oral tramadol in addition to local infiltration of lignocaine (Arm $\mathrm{T}$ ), local application of EMA cream in addition to local infiltration of lignocaine (Arm E), and only to local infiltration of lignocaine (Arm L). Randomization was done using lottery method. Based on the previous studies using tramadol or EMA cream for preventing pain with BMAB, a minimum sample size of 75 in each arm was determined. We decided to have 100 patients in each arm.

The procedures were done by one of the Medical oncology residents. The assessment of pain and anxiety was done by the investigator who did not know to which arm the patients were randomized.

All BMAB were done from posterior superior iliac crest. Standard procedure (Arm L) included infiltration of $10 \mathrm{~mL}$ of $2 \%$ lignocaine into skin, subcutaneous tissue, and periosteum around the BMAB needle insertion site 10 minutes before the start of the procedure. Bone marrow aspiration was done using Jamshidi needle with aspiration of adequate amount of BM aspirate followed by bone marrow biopsy from the same site with the removal of adequate amount of bone marrow core.

In the tramadol arm (Arm T), oral tramadol $50 \mathrm{mg}$ was given 1 hour prior to the procedure along with local lignocaine infiltration as in the standard procedure. In the other two arms, similar appearing placebo was given orally 1 hour before the procedure.

In the EMA cream arm (Arm E), $2.5 \mathrm{~g}$ of EMA cream was applied along with an occlusive dressing over the posterior superior iliac spine 1 hour prior to the procedure along with local lignocaine infiltration as in the standard procedure. In the other two arms, a similar appearing cream was applied along with an occlusive dressing on the posterior superior iliac spine 1 hour prior to the procedure.

Basic details of all the patients undergoing BMAB were noted like age, sex, presence of any comorbidities, Eastern Oncology Group (ECOG) performance status, education level (whether able to interpret the written questionnaire on their own or not), body mass index, suspected or proved diagnosis, and whether it was a repeat procedure.

Pain intensity was noted according to FACES pain scale, a visual scale with the range 0 to $10 .^{2}$ This was noted at the time of local infiltration of lignocaine, at the time of BMAB and at 15 minutes post procedure (after application of pressure bandage). Anxiety was assessed using numerical rating score on a scale of 0 to $10 .^{3}$ 
Table 1 Basic details of patients undergoing BMAB

\begin{tabular}{|l|l|l|l|l|l|l|}
\hline Arm & Tramadol & EMA & Lignocaine only & Total & $\begin{array}{l}\text { p-Value for relation to } \\
\text { moderate/severe pain }\end{array}$ \\
\hline \multirow{2}{*}{ No. of cases } & Male & 100 & 100 & 100 & 300 & \\
\cline { 2 - 7 } Sex & Female & 60 & 69 & 64 & $193(64.3 \%)$ & NS \\
\hline \multirow{2}{*}{ Age $>50$} & 40 & 31 & 36 & $107(35.7 \%)$ & NS \\
\hline PS $>3$ & 32 & 29 & 40 & $101(34 \%)$ & 0.017 \\
\hline BMI $>30$ & 4 & 6 & 6 & $16(5.6 \%)$ & 0.05 \\
\hline Educated & 7 & 8 & 5 & $20(6.7 \%)$ & 0.05 \\
\hline Repeat procedure & 84 & 81 & 78 & $243(81 \%)$ & NS \\
\hline Duration $>10$ min & 34 & 27 & 32 & $93(31 \%)$ & NS \\
\hline $\begin{array}{l}\text { Primary } \\
\text { diagnosis }\end{array}$ & Acute leukemia & 68 & 8 & 12 & $29(9.7 \%)$ & 0.04 \\
\cline { 2 - 7 } & CML & 16 & 66 & 71 & $205(68.3 \%)$ & 0.05 \\
\cline { 2 - 7 } & Lymphoma & 11 & 20 & 17 & $53(17.7 \%)$ & NS \\
\cline { 2 - 7 } & Others & 5 & 8 & 6 & $25(8.3 \%)$ & NS \\
\hline
\end{tabular}

Abbreviations: BMAB, bone marrow aspiration and biopsy; BMI, body mass index; CML, chronic myelogenous leukemia.

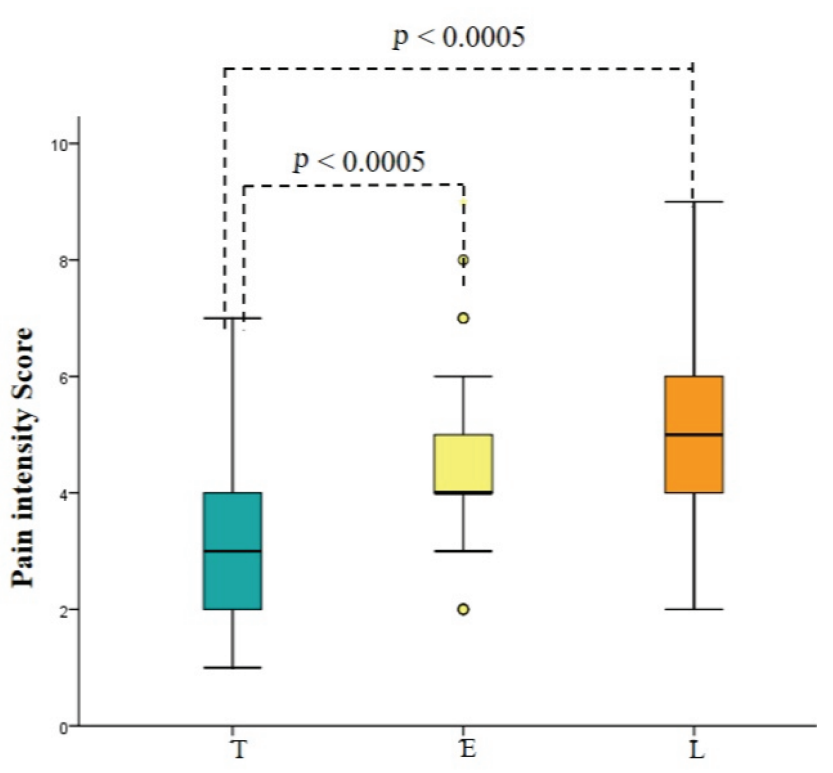

Fig. 1 Box plot comparing pain intensity during BMAB in tramadol arm with the other arms. BMAB, bone marrow aspiration and biopsy.

The duration of the procedure was recorded as the time from the BMAB needle piercing the skin to the application of pressure bandage.

Patients were observed for an hour post procedure and were told to specifically report any vomiting, nausea, dizziness, and sedation. They were instructed to report if they developed any discomfort later also.

Statistical analysis was done using the latest SPSS version available and a - value of less than 0.05 was considered significant.

\section{Results}

A total of 300 cases were analyzed with 100 cases in each of the three arms. The details are mentioned in - Table 1.
Pain

Pain at various points of procedure is recorded in - Table 2.

The mean pain intensity during aspiration/biopsy part was significantly lower $(p<0.001)$ in the tramadol arm compared with the EMA and the lignocaine only arm-Fig. 1 . The percentage of patients with moderate or severe pain (defined as a score of 4 or more) was also significantly lower in the tramadol arm compared with the EMA and the lignocaine only arm. The mean pain intensity during skin puncture was significantly lower in the EMA arm compared with the tramadol and the lignocaine only arm. There was no difference in the mean pain intensity post procedure in any of the three arms.

We also did a univariate analysis to see which of the patient- or procedure-related factors were associated with moderate or severe pain. The $p$-values of the same are mentioned in - Table 1 .

\section{Anxiety}

There was no significant difference in mean anxiety score between either of the three arms. There was no correlation between patients' anxiety scores and the intensity of pain severity.

\section{Adverse Effects}

Tramadol was well tolerated. Five patients experienced dizziness and three patients experienced sedation with tramadol. Whereas in the EMA and the lignocaine local infiltration only arm, three patients each had dizziness while no patients experienced sedation.

\section{Discussion}

A few studies have tested the use of opioids, benzodiazepines alone, or in combination for the prevention of BMAB pain with mixed results and without any clarity on the benefit of any of the drugs. EMA cream has also been tried in adults 
Table 2 Pain intensity and anxiety in the three arms

\begin{tabular}{|l|l|l|l|l|l|}
\hline Arm & Tramadol & EMA & Lignocaine only & Total \\
\hline \multirow{2}{*}{$\begin{array}{l}\text { Mean pain } \\
\text { intensity }\end{array}$} & Local anesthesia & $1.1 \pm 0.3$ & $0.8 \pm 0.5$ & $1.1 \pm 0.3$ & $1 \pm 0.4$ \\
\cline { 2 - 6 } & Periosteal puncture & $1.9 \pm 0.4$ & $2 \pm 0.4$ & $2.1 \pm 0.4$ & $2 \pm 0.4$ \\
\cline { 2 - 6 } & Aspiration/biopsy & $3.4 \pm 1.2$ & $4.4 \pm 1.4$ & $4.7 \pm 1.5$ & $4.1 \pm 1.5$ \\
\cline { 2 - 6 } & Residual & $1.1 \pm 0.4$ & $1.2 \pm 0.4$ & $1.2 \pm 0.5$ & $1.1 \pm 0.4$ \\
\hline \% Patients with moderate/severe pain & 45 & 77 & 82 & 68 \\
\hline \multicolumn{2}{|l|}{ Anxiety score } & $1.9 \pm 0.4$ & $2 \pm 0.4$ & $2.1 \pm 0.5$ & $2 \pm 0.4$ \\
\hline
\end{tabular}

Abbreviation: EMA, eutectic mixture of local anesthetics.

and children for BMAB prevention but without any significant results.

Kuivalainen tried sublingual fentanyl 100 or $200 \mu$ g before the procedure for prevention of BMAB-associated pain but found no benefit of adding fentanyl compared with placebo while adverse effects were more in the fentanyl arm. Inadequate amount of time before the procedure was postulated as a possible cause of lack of benefit. ${ }^{3}$

Vanhelleputte et al used oral tramadol $50 \mathrm{mg}$ or placebo 1 hour before BMAB for pain prevention. They found a significantly lower pain intensity on the visual analogue scale of $16.6 \pm 3 \mathrm{~mm}$ in Tramadol arm compared with $28.8 \pm 3.4 \mathrm{~mm}$ in the placebo arm. The percentage of patients experiencing at least moderate pain was reduced from $40 \%$ in the placebo arm to $20 \%$ in the tramadol arm. ${ }^{8}$

Talamo et al used a combination of acetaminophen $650 \mathrm{mg}$ plus oxycodone $10 \mathrm{mg}$ plus lorazepam $2 \mathrm{mg}$, all given orally half an hour before BMAB. Seventy-eight percent of patients in the combination arm had lower pain compared with $64 \%$ in the placebo arm which they reported to be statistically significant but clinically not meaningful due to vast majority of patients still experiencing significant pain. ${ }^{9}$

Wolanskyj et al used a combination of lorazepam $2 \mathrm{mg}$ plus hydromorphone $2 \mathrm{mg}$ orally 1 hour before BMAB but could not demonstrate any significant reduction in pain. ${ }^{10}$

Dunlop et al had used the same combination of lorazepam and hydromorphone 90 minutes before BMAB with a weight-based dose and found significant reduction in the percentage of patients experiencing moderate/severe pain. ${ }^{11}$

Holdsworth et al compared the use of propofol/fentanyl general anesthesia versus only EMA cream versus EMA cream plus midazolam for BMAB pain prevention in children and found general anesthesia to be significantly effective in reducing pain compared with EMA and EMA/midazolam. ${ }^{12}$

$\mathrm{Ng}$ et al used EMA along with patient-controlled analgesia with alfentanil and local anesthesia for reducing pain during bone marrow harvesting but they did not compare the effect of addition of EMA on pain reduction. ${ }^{13}$

In our study, we found a significant reduction in the pain severity with the use of tramadol $50 \mathrm{mg}$ per oral along with local lignocaine infiltration compared with lignocaine local infiltration alone or EMA cream added to local lignocaine infiltration prior to BMAB. Also, the percentage of patients who experienced moderate or severe pain with BMAB was reduced significantly in the tramadol arm.
In our study, mean pain intensity was higher compared with other studies probably because all of our patients underwent bone marrow core biopsy in addition to bone marrow aspiration. And this may also have contributed to our patients having significant benefit in pain reduction compared with some of the other negative studies. ${ }^{14}$

In various studies, different factors have been found to correlate with increased pain severity with BMAB despite various interventions. Some of the factors found by them to be associated with increased probability of experiencing higher pain are high BMI, increased duration of the procedure, younger age, and anxious patients. ${ }^{1}$ In our study, we studied various variables and found the following factors to be associated significantly with higher pain: ECOG performance status $\geq 3$, age $\geq 50$, duration of $B M A B>10$ minutes, $B M I \geq 30$, and a provisional/confirmed diagnosis of acute leukemia.

\section{Conclusion}

Since pain intensity is subjective, an objective benefit in pain reduction with BMAB is difficult to measure and hence may be debatable. But we demonstrated a small but significant benefit of adding oral tramadol to local lignocaine infiltration. Further, larger studies in different patient populations may be done to confirm this benefit and its subsequent incorporation into widespread clinical practice.

Note

Presented at ESMO Asia conference, Singapore on November 22 to November 24, 2019. by the corresponding author in the poster presentation category.

\section{Conflict of Interest}

None declared.

\section{Acknowledgment}

Family members of all authors. And all the patients who consented for the study for the advancement of science. To all the residents and sisters of Medical Oncology Department at our institute for co-operating and helping with this study.

\section{References}

1 Zahid MF. Methods of reducing pain during bone marrow biopsy: a narrative review. Ann Palliat Med 2015;4(4):184-193 
2 Gendron N, Zia Chahabi S, Poenou G, et al. Pain assessment and factors influencing pain during bone marrow aspiration: a prospective study. PLoS One 2019;14(8):e0221534

3 Kuivalainen A-M. Pain and associated procedural anxiety in adults undergoing bone marrow aspiration and biopsy: therapeutic efficacy and feasibility of various analgesics. 2015. Available at: https://researchportal.helsinki.fi/en/publications/pain-and-associated-procedural-anxiety-in-adults-undergoing-bone-. Accessed December 5, 2019

4 CerchioneC, MartinelliG, Catalano L, etal.Combined oraladministration of analgesia and anxiolysis for pain associated with bone marrow aspiration and biopsy. J Clin Oncol 2019;37(suppl 15): e18313.10.1200/JCO.2019.37.15_suppl.e18313

5 Milligan DW, Howard MR, Judd A. Premedication with lorazepam before bone marrow biopsy. J Clin Pathol 1987;40(6):696-698

6 Watmough S, Flynn M. A review of pain management interventions in bone marrow biopsy.J Clin Nurs 2011;20(5-6):615-623

7 Buckley MM, Benfield P. Eutectic lidocaine/prilocaine cream. A review of the topical anaesthetic/analgesic efficacy of a eutectic mixture of local anaesthetics (EMLA) Drugs 1993;46(1):126-151

8 Vanhelleputte P, Nijs K, Delforge M, Evers G, Vanderschueren S. Pain during bone marrow aspiration: prevalence and prevention. J Pain Symptom Manage 2003;26(3):860-866
9 Talamo G, Liao J, Bayerl MG, Claxton DF, Zangari M. Oral administration of analgesia and anxiolysis for pain associated with bone marrow biopsy. Support Care Cancer 2010;18(3):301-305

10 Wolanskyj AP, Schroeder G, Wilson PR, Habermann TM, Inwards DJ, Witzig TE. A randomized, placebo-controlled study of outpatient premedication for bone marrow biopsy in adults with lymphoma. Clin Lymphoma 2000;1(2):154-157

11 Dunlop TJ, Deen C, Lind S, Voyle RJ, Prichard JG, Use of combined oral narcotic and benzodiazepine for control of pain associated with bone marrow examination. South Med J. 1999 May;92(5):477-80. doi: 10.1097/00007611-19990500000005. PMID: 10342892.

12 Holdsworth MT, Raisch DW, Winter SS, et al. Pain and distress from bone marrow aspirations and lumbar punctures. Ann Pharmacother 2003;37(1):17-22

$13 \mathrm{Ng} \mathrm{HN}$, Sim KM, Boey SK. Bone marrow harvesting using EMLA (eutectic mixture of local anaesthetics) cream, local anaesthesia and patient-controlled analgesia with alfentanil. Bone Marrow Transplant 1999;23(9):941-945

14 Holdsworth MT, Raisch DW, Winter SS, et al. Pain and distress from bone marrow aspirations and lumbar punctures; 2003. Available at: https://journals.sagepub.com/doi/abs/10.1345/ aph.1C088?rfr_dat=cr_pub\%3Dpubmed\&url_ver=Z39.88$2003 \&$ rfr_id=ori\%3Arid\%3Acrossref.org\&journalCode=aopd. Accessed December 7, 2019 